\title{
Bartonella quintana and Typhus Group Rickettsiae Exposure among Homeless Persons, Bogotá, Colombia
}

\section{Álvaro A. Faccini-Martínez, Andrea C. Márquez, Diana M. Bravo-Estupiñan, Omar-Javier Calixto, Christian A. López-Castillo, Carlos A. Botero-García, Marylin Hidalgo, Claudia Cuervo}

In 2015, we investigated Bartonella quintana and typhus group rickettsiae in body lice from homeless persons in Bogotá, Colombia. We found $B$. quintana-infected body lice and seroprevalence of this microorganism in $19 \%$ of homeless persons and typhus group rickettsiae in $56 \%$. Public health professionals should start preemptive measures and active vector control.

$\mathrm{H}$ omeless persons make up part of the population at highest risk for infectious diseases because of factors such as deficient hygiene habits, infrequent washing and changing clothes, and overcrowding (1). Within this group, vector-borne diseases caused by bacteria of the genera Bartonella, Rickettsia, and Borrelia are of great importance; louseborne $B$. quintana is the main microorganism associated with infections in homeless persons (1). In Colombia, the presence of $R$. prowazekii, a typhus group rickettsiae (TGR), in lice and related human infections in Bogotá was evident only during 1918-1922 and 1941 (2). We investigated the presence of B. quintana and TGR in body lice collected from homeless persons in Bogotá and these persons' exposure to such microorganisms.

\section{The Study}

The Research and Ethics Committees of the Facultad de Ciencias of the Pontificia Universidad Javeriana (Bogotá, Colombia) approved this study (February 14, 2013). All participants read, accepted, and signed the informed consent form.

Author affiliations: Universidade Federal do Espírito Santo, Vitória, Espírito Santo, Brazil (Á.A. Faccini-Martínez); Pontificia Universidad Javeriana, Bogotá, Colombia (A.C. Márquez, D.M. Bravo-Estupiñan, M. Hidalgo, C. Cuervo); Hasselt University, Brussels, Belgium (O.-J. Calixto); Asociación Colombiana de Infectología, Bogotá (C.A. López-Castillo); Hospital Militar Central, Bogotá (C.A. Botero-García)

DOI: https://doi.org/10.3201/eid2311.170341
During May-September 2015, we enrolled a total of 153 persons from a homeless shelter in Bogotá in the study and obtained serum samples from each person. We also collected lice from participants' clothing or body (below the neck) and considered these lice a positive indication of body louse infestation. Of the study participants, 132 were men, 17 were women, and 4 were transgender (for the study, people who had a gender identity or gender expression that differs from their assigned sex). Participants' mean age was $39.6(\mathrm{SD} \pm 11.65)$ years. Eighteen (11.7\%) were infested with body lice; lice were preliminarily identified as Pediculus humanus humanus, according to standard taxonomic keys (3), and all had the same light color (Figure 1).

To detect specific IgG to Bartonella spp. (B. quintana antigen) and TGR ( $R$. typhi antigen) in serum samples, we used commercially sourced indirect fluorescent antibody (IFA) kits (Bartonella IFA IgG and Rickettsia IFA IgG; Focus Technologies, Cypress, CA, USA). We screened serum at a dilution of 1:64. Among the 153 study participants, 29 (19.0\%) had IgG that reacted exclusively against Bartonella spp. at a titer of $\geq 64$, and 86 (56.2\%) had IgG that reacted exclusively against TGR at a titer of $\geq 64$. Twenty (13.1\%) participants had IgG against both Bartonella spp. and TGR.

For identification of Bartonella spp. and Rickettsia spp. from louse samples, we organized 201 body lice into 39 pools and extracted DNA from each pool (DNeasy Blood and Tissue; QIAGEN, Valencia, CA, USA). We screened all louse pools by standard PCR for Bartonella spp. (citrate synthase gene $[$ gltA] and 16S-23S rRNA intergenic transcribed spacer region [ITS-1]) and Rickettsia spp. (gltA, 16S RNA, and ompB rickettsial genes) as described (4). Eleven (28\%) louse pools were positive for Bartonella spp., of which 7 were positive for glt $A, 10$ were positive for ITS-1, and 6 were positive for both genes. We found no evidence of Rickettsia spp. infection in body lice. For Bartonella spp., sequence of the ITS-1 fragment amplified by standard PCR and phylogenetic analysis with maximum-likelihood method and 1,000 bootstrap replicates, performed using MEGA software version 6 (5), confirmed the bacteria as $B$. quintana (GenBank accession no. KY605045) (Figure 2, panel A).

To examine the mitochondrial clade in $P . h$. humanus captured in this study, we tested DNA samples from 2 

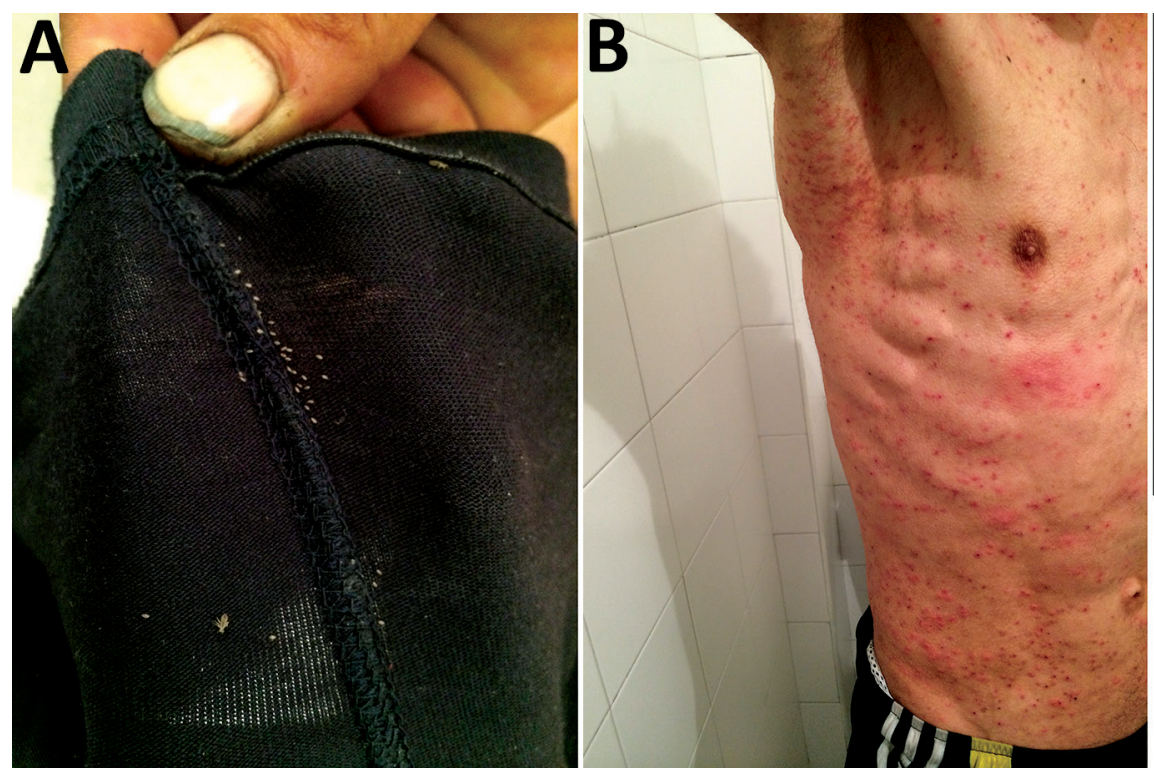

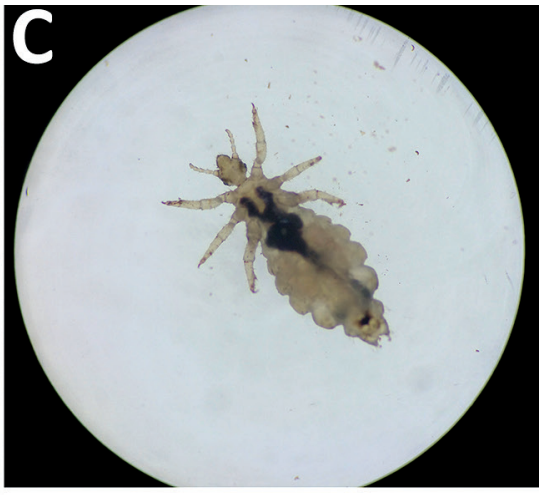

Figure 1. Homeless man infested by body lice, Bogotá, Colombia, 2015. A) Body lice and eggs in clothing seams. B) Pruritic and scratching lesions on the man's body. C) Adult female body louse collected from clothing. randomly selected lice and PCR amplified the $c y t b$ genes as reported (6). We compared the cytb sequences obtained in our study with known head and body louse sequences from the 5 P. h. humanus clades (A-E) (6). Phylogenetic analysis using the maximum-likelihood method demonstrated that the 2 sequences obtained in our study belong to clade A (GenBank accession nos. KY605043 and KY605044) (Figure 2, panel B).

\section{Conclusions}

Our study demonstrates evidence of infestation by the body louse ( $P$. h. humanus) infected with $B$. quintana and exposure to TGR in homeless persons in Bogotá. The rate of body louse infestation in the studied population $(11.7 \%)$ was within the range reported elsewhere $(7 \%$ $30 \%)(1,7)$, confirming that homeless persons are among the population groups most vulnerable to parasitism by this arthropod and associated infectious agents (1). We also found seroprevalence for Bartonella spp. (19.0\%) in line with the range reported in other studies worldwide $(0.4 \%-62 \%)(8,9)$. Although cross-reactions in the IFA between different Bartonella species are possible (highlighting those associated with homeless persons: $B$. quintana, B. elizabethae, and B. henselae) $(8,9)$, we consider that the seroprevalence detected in homeless persons in Bogotá probably is due to B. quintana because it is the microorganism most frequently associated with homeless persons (1), and we detected it in $28.2 \%$ of body lice collected from persons sampled, again in agreement with previous studies (1.4\%-94\%) (10).

On the other hand, the level of seropositivity against TGR found in our study (56.2\%) was considerably higher than levels reported in previous studies $(\approx 0.54 \%-22 \%)(9)$. We were not able to perform a Western blot-associated cross-adsorption test to distinguish the specific Rickettsia species involved in the TGR-positive serum (11). However, we consider that $R$. typh $i$ was probably the predominant species responsible for the seropositivity for the following reasons: $R$. prowazekii was not detected in collected body lice; infection with $R$. typhi is frequent in homeless persons (11); and no records from healthcare government entities in Colombia, whether local (Bogotá) or national, suggest the occurrence in this population of febrile illness with high death rates, which would be more compatible with the epidemiology of epidemic typhus ( $R$. prowazekii infection) than with murine typhus ( $R$. typhi infection) (12). Although human body lice are not clearly identified vectors of $R$. typhi, it seems that under certain circumstances they could transmit $R$. typhi (13), as well as other rickettsiae (14). More work is needed to identify properly the Rickettsia and Bartonella species involved in this antibody prevalence.

We identified the lice collected from homeless persons in Bogotá as belonging to the haplogroup/clade A, which is distributed worldwide and comprises $P$. h. humanus and $P$. h. capitis (6). In our study, given that lice were collected from clothes and body areas below the neck, they probably were $P$. h. humanus. Nevertheless, molecular determination by PCR using the Phum_PHUM540560 gene currently is the only way to distinguish body and head lice (15).

Our study is subject to several limitations. The detection of Bartonella spp. and Rickettsia spp. was based on standard PCR and not on real-time PCR, which is more sensitive. As a result, some samples could have tested negative because of low DNA load. Moreover, we did not test serum or blood samples by molecular assays, nor perform cultures to isolate infectious agents from the lice. Nonetheless, our study results should encourage public health professionals in 

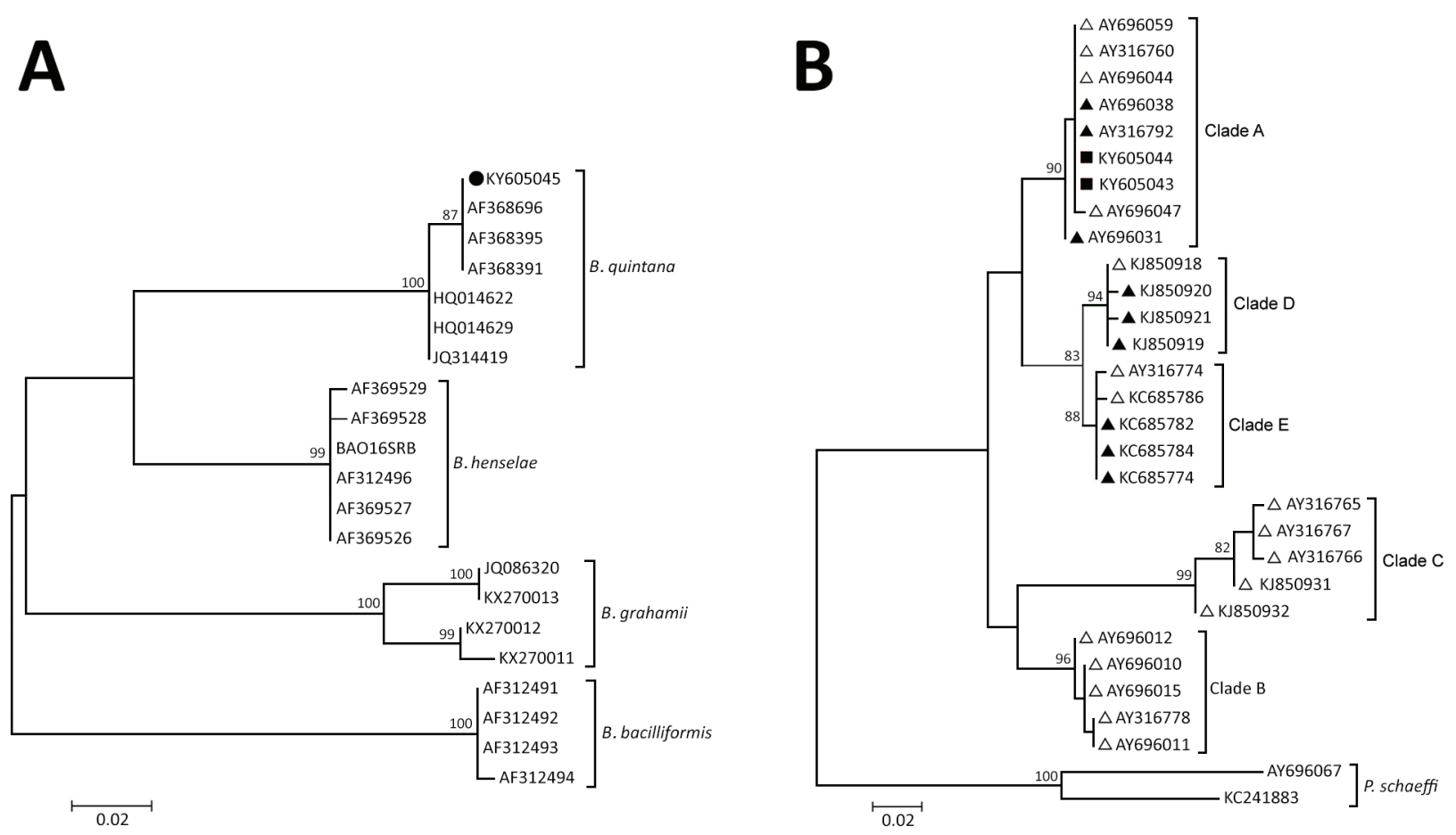

Figure 2. Maximum-likelihood (ML) analyses of intergenic transcribed spacer 1 genes of Bartonella spp. and of the Pediculus humanus humanus louse mitochondrial cytochrome b (cytb) gene. A) Bartonella spp. analysis. The tree with the highest log likelihood is shown, and ML bootstrap values $>80$ are indicated at each node. The tree is drawn to scale. Sequences are indicated by GenBank accession number; solid circle indicates the sequence retrieved in this study. The Bartonella species is indicated to the right of each branch. B) $P$. h. humanus analysis. The tree with the highest log likelihood is shown, and ML bootstrap values are located above the node. The tree is drawn to scale. The body louse sequences (solid triangles), head louse sequences (open triangles), and GenBank accession numbers are indicated. Cytochrome b sequences from Pediculus schaeffi were used as outgroups. Solid squares indicate the sequences retrieved in this study. The mitochondrial clade is indicated to the right of each branch. Scale bars indicate nucleotide substitutions per site.

Bogotá to start preemptive measures and active vector control (delousing and ivermectim treatment) (1), conduct future research evaluating the clinical characteristics of Bartonella and Rickettsia infections in homeless persons in Bogotá, confirm circulation of specific species of these microorganisms, and screen for Bartonella spp. endocarditis by blood culture among homeless persons who have high antibody titers.

\section{Acknowledgments}

We thank the homeless persons who participated in our study. We also thank the Secretaria Distrital de Integración Social, Bogotá, and Secretaria Distrital de Salud, Bogotá for providing access to shelters. We express our appreciation to Lesley Bell-Sakyi for reviewing the English language of this manuscript.

This work was supported by Vicerrectoria de Investigación, Pontificia Universidad Javeriana, Bogotá, (research project no. 00005552).

Dr. Faccini-Martínez is a PhD student in the Postgraduate Program in Infectious Diseases, Health Science Center, Universidade Federal do Espírito Santo, Vitória, ES,
Brazil. His primary research interests include zoonotic and vectorborne diseases.

\section{References}

1. Badiaga S, Raoult D, Brouqui P. Preventing and controlling emerging and reemerging transmissible diseases in the homeless. Emerg Infect Dis. 2008;14:1353-9. http://dx.doi.org/10.3201/ eid1409.080204

2. Faccini-Martínez ÁA, Botero-García CA, Hidalgo M. Contributions to rickettsioses research in Colombia (19171943), Luis B. Patiño Camargo. Rev Inst Med Trop Sao Paulo. 2016;58:33. http://dx.doi.org/10.1590/S1678-9946201658033

3. Bonilla DL, Durden LA, Eremeeva ME, Dasch GA. The biology and taxonomy of head and body lice-implications for louseborne disease prevention. PLoS Pathog. 2013;9:e1003724. http://dx.doi.org/10.1371/journal.ppat.1003724

4. Roux V, Raoult D. Body lice as tools for diagnosis and surveillance of reemerging diseases. J Clin Microbiol. 1999;37:596-9.

5. Tamura K, Stecher G, Peterson D, Filipski A, Kumar S. MEGA6: Molecular Evolutionary Genetics Analysis version 6.0. Mol Biol Evol. 2013;30:2725-9. http://dx.doi.org/10.1093/molbev/mst197

6. Ashfaq M, Prosser S, Nasir S, Masood M, Ratnasingham S, Hebert PD. High diversity and rapid diversification in the head louse, Pediculus humanus (Pediculidae: Phthiraptera). Sci Rep. 2015;5:14188. http://dx.doi.org/10.1038/srep14188 
7. Bonilla DL, Cole-Porse C, Kjemtrup A, Osikowicz L, Kosoy M. Risk factors for human lice and bartonellosis among the homeless, San Francisco, California, USA. Emerg Infect Dis. 2014;20:164551. http://dx.doi.org/10.3201/eid2010.131655

8. Ehrenborg C, Byström R, Hjelm E, Friman G, Holmberg M. High Bartonella spp. seroprevalence in a Swedish homeless population but no evidence of trench fever. Scand J Infect Dis. 2008;40:208-15. http://dx.doi.org/10.1080/ 00365540701632972

9. Leibler JH, Zakhour CM, Gadhoke P, Gaeta JM. Zoonotic and vector-borne infections among urban homeless and marginalized people in the United States and Europe, 1990-2014. Vector Borne Zoonotic Dis. 2016;16:435-44. http://dx.doi.org/ 10.1089/vbz.2015.1863

10. Fournier PE, Ndihokubwayo JB, Guidran J, Kelly PJ, Raoult D. Human pathogens in body and head lice. Emerg Infect Dis. 2002;8:1515-8. http://dx.doi.org/10.3201/eid0812.020111

11. Badiaga S, Benkouiten S, Hajji H, Raoult D, Brouqui P. Murine typhus in the homeless. Comp Immunol Microbiol Infect Dis. 2012;35:39-43. http://dx.doi.org/10.1016/ j.cimid.2011.09.008
12. Brouqui P, Raoult D. Arthropod-borne diseases in homeless. Ann N Y Acad Sci. 2006;1078:223-35. http://dx.doi.org/10.1196/annals. 1374.041

13. Houhamdi L, Fournier PE, Fang R, Raoult D. An experimental model of human body louse infection with Rickettsia typhi. Ann N Y Acad Sci. 2003;990:617-27. http://dx.doi.org/10.1111/j.1749-6632.2003. tb07436.x

14. Houhamdi L, Raoult D. Experimentally infected human body lice (Pediculus humanus humanus) as vectors of Rickettsia rickettsii and Rickettsia conorii in a rabbit model. Am J Trop Med Hyg. 2006;74:521-5.

15. Drali R, Boutellis A, Raoult D, Rolain JM, Brouqui P. Distinguishing body lice from head lice by multiplex real-time PCR analysis of the Phum_PHUM540560 gene. PLoS One. 2013;8:e58088. http://dx.doi.org/10.1371/journal.pone.0058088

Address for correspondence: Claudia Cuervo, Grupo de Enfermedades Infecciosas, Departamento de Microbiología, Facultad de Ciencias, Pontificia Universidad Javeriana, Carrera 7 \#43-82, Edificio 52, Oficina 608, Bogotá, DC, Colombia; email: claudia.cuervo@javeriana.edu.co

\section{August 2016: Parasitology}

- Coinfections with Visceral Pentastomiasis, Democratic Republic of the Congo

- Probable Rabies Virus Transmission through Organ Transplantation, China, 2015

- Microgeographic Heterogeneity of Border Malaria During Elimination Phase, Yunnan Province, China

- Human Babesiosis, Bolivia, 2013

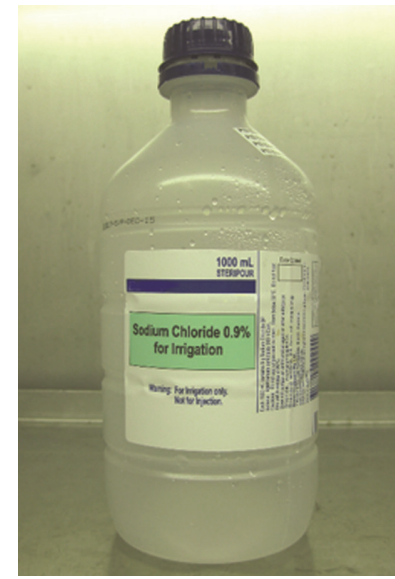

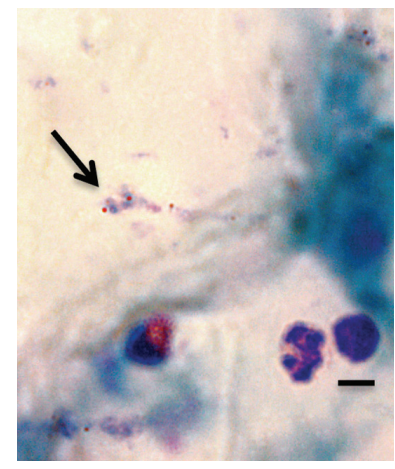

- Virulence and Evolution of West Nile Virus, Australia, 1960-2012

- Multilocus Sequence Typing Tool for Cyclospora cayetanensis

- Phylogeographic Evidence for Two Genetically Distinct Zoonotic Plasmodium knowlesi Parasites, Malaysia

- Hemolysis after Oral Artemisinin Combination Therapy for Uncomplicated Plasmodium falciparum Malaria
- Middle East Respiratory Syndrome Coronavirus Transmission in Extended Family, Saudi Arabia, 2014

- Exposure-Specific and Age-Specific Attack Rates for Ebola Virus Disease in EbolaAffected Households, Sierra Leone

- Outbreak of Achromobacter xylosoxidans and Ochrobactrum anthropi Infections after Prostate Biopsies, France, 2014

- Lyssavirus in Indian Flying Foxes, Sri Lanka

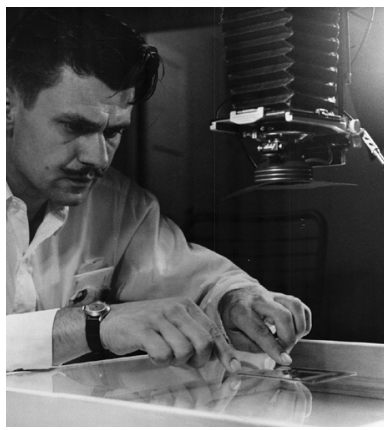

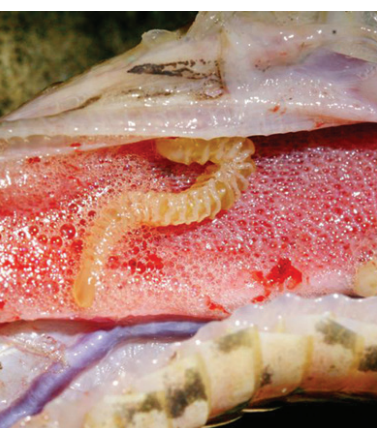

- Possible Role of Fish and Frogs as Paratenic Hosts of Dracunculus medinensis, Chad

- Importation of Hybrid Human-Associated Trypanosoma cruzi Strains of Southern South American Origin, Colombia

- Survival and Growth of Orientia tsutsugamushi in Conventional Hemocultures

- Borrelia miyamotoi Infection in Patients from the Upper Midwestern United States, 2014-2015 